ÉGYPTE

monde arabe

\section{Égypte/Monde arabe}

26 | 1996

Mutations

\title{
La Nahda ou le rêve de la nation égyptienne de Muhammad 'Ali à Gamal Abdel Nasser
}

\section{Hossam 'Issa}

\section{(2) OpenEdition}

1 Journals

Édition électronique

URL : https://journals.openedition.org/ema/1480

DOI : $10.4000 /$ ema. 1480

ISSN : 2090-7273

\section{Éditeur}

CEDEJ - Centre d'études et de documentation économiques juridiques et sociales

\section{Édition imprimée}

Date de publication : 30 juin 1996

Pagination : 173-184

ISSN : 1110-5097

\section{Référence électronique}

Hossam 'Issa, "La Nahda ou le rêve de la nation égyptienne de Muhammad 'Ali à Gamal Abdel Nasser », Égypte/Monde arabe [En ligne], 26 | 1996, mis en ligne le 08 juillet 2008, consulté le 07 juillet 2022. URL : http://journals.openedition.org/ema/1480 ; DOI : https://doi.org/10.4000/ema.1480

Ce document a été généré automatiquement le 7 juillet 2022.

Tous droits réservés 


\title{
La Nahda ou le rêve de la nation égyptienne de Muhammad 'Ali à Gamal Abdel Nasser
}

\author{
Hossam 'Issa
}

\section{NOTE DE L'ÉDITEUR}

Professeur à la faculté de Droit de 'Ayn Shams, Hossam 'Issa est par ailleurs une grande figure nassérienne et un des leaders du nouveau parti nassérien, constitué en 1992. Il est l'auteur de nombreux ouvrages, notamment sur le transfert de technologies, les rapports Nord-Sud, le Japon... Il a rédigé le présent texte à l'occasion du vingtcinquième anniversaire de la disparition de Gamal Abdel Nasser.

1 Comment se fait-il que le monde soit devenu l'enfer des croyants et le paradis des incroyants? Telle est la question qui a hanté le XIX ${ }^{e}$ siècle après que le règne de Muhammad 'Ali eut succombé aux déprédations commises par les armées, les flottes et les commerçants de l'Occident. Posée en ces termes, dans l'esprit religieux de l'époque, elle peut paraître naïve. Elle a pourtant sous-tendu toute la pensée des artisans de la Nahda (« renaissance »), de Tahtâwi à Jamâl al-Dîn al-Afghâni et Muhammad 'Abduh, en passant par 'Abd Allah al-Nadîm. En effet, il s'agissait pour eux de déterminer les voies qui mèneraient à la Nahda en s'interrogeant, d'une part, sur les causes du retard de l'Orient musulman en général et de l'Égypte en particulier et, d'autre part, sur les raisons de l'avancée de l'Occident et les moyens de le rattraper.

Dès le départ, comme le note à juste titre Anouar Abdel Malek, la question était d'ordre politique, et si, très vite, de multiples réponses ont été avancées, toutes constituaient en quelque sorte un commentaire de l'expérience de Muhammad 'Ali. C'était la référence incontournable des penseurs de la Nahda. Cela est particulièrement clair chez Tahtâwi, que l'on considère généralement comme le père spirituel des divers courants 
de la pensée moderne en Égypte et, en particulier, du «libéralisme» et du « radicalisme».

3 Même le réformisme musulman d'un Muhammad 'Abduh, malgré des divergences de fond évidentes, n'a pu échapper à l'empreinte qu'a laissée l'expérience politique de Muhammad 'Ali dans l'esprit des Égyptiens : vers la fin de sa vie, tout son discours se polarisa sur l'importance de la science et de l'éducation, présentées comme le secret de la réussite et de la force de l'Occident.

De fait, l'expérience de Muhammad 'Ali - dont Marx disait qu'il était le seul personnage de l'Empire ottoman a avoir eu un cerveau à la place du tarbouche - représente bien la première tentative de Nahda dans notre histoire moderne. Au système mamelouk semiféodal, que Samîr Amîn a qualifié de «mode de production tributaire », Muhammad 'Ali substitua en effet la force d'un État centralisé contrôlant tous les centres de décision politique et économique, de sorte qu'à la faveur de l'étatisation de l'agriculture, de l'industrie et du commerce, l'Égypte fut la première formation sociale en Orient à mener à bien une entreprise de construction nationale indépendante, plus d'un demisiècle avant l'ère du Meiji au Japon. Cette entreprise fut marquée avant tout par la mise en place d'une industrie moderne, notamment dans le domaine militaire, le développement des systèmes d'irrigation et de l'agriculture, la réforme de la législation fiscale, la diffusion de l'enseignement et la constitution d'une puissante armée nationale garante de la sauvegarde de ces réalisations.

Parallèlement à la Nahda économique, un vaste mouvement de renaissance culturelle vit le jour, qui resta associé, dans l'esprit des Égyptiens, à des noms de penseurs comme Hasan al-'Attâr ou Rifâ'a al-Tahtâwi. Cette renaissance se manifesta notamment par un renouveau des études sur la langue arabe : on chercha ainsi les moyens stylistiques qui permettraient d'adapter l'arabe aux exigences de l'édification du nouvel État. En outre, les sciences historiques prirent un nouvel essor, ainsi que la géographie, dont le renouvellement allait de pair avec la modernisation de la toute récente armée nationale.

6 Sur ce, survint le Traité de Londres de 1840, que les forces européennes, menées par la Grande-Bretagne, imposèrent par les armes à Muhammad 'Ali et en vertu duquel l'Égypte s'engageait à ouvrir son marché et à supprimer les monopoles de l'État sur le commerce. C'était le coup d'envoi d'une nouvelle période dans l'histoire de l'Égypte, qui consacra l'abolition du centralisme de l'État et son renoncement à jouer un rôle dans l'entreprise de la Nahda. Au nom de la «liberté du commerce», le marché économique égyptien fut ainsi livré à l'Occident et le vaste projet d'industrialisation entrepris par Muhammad 'Ali s'effondra sous les coups de la concurrence étrangère : en très peu d'années, il ne resta plus de ce projet que quelques machines couvertes de rouille abandonnées dans des bâtiments en ruine.

7 À aucun moment la Grande-Bretagne ne cacha son intention de liquider le pouvoir de Muhammad 'Ali car, selon l'expression du premier ministre de l'époque, Palmerston, il était hors de question de laisser la route de l'Inde sous le contrôle d'un dirigeant arabe aussi puissant que Muhammad 'Ali, à plus forte raison si celui-ci était en bons termes avec la France.

8 L'État centralisé ayant été dessaisi de ses monopoles et ayant perdu toute influence sur le processus social, l'Égypte se vit intégrée au marché international des capitaux et des marchandises et confrontée par là même à l'industrie et au commerce européens. À l'ombre du système des concessions étrangères, des aventuriers et spéculateurs venus 
de toutes parts affluèrent vers cette nouvelle Californie qu'était l'Égypte, non pas pour son or mais bien pour son coton, ce coton qui faisait tant défaut à l'Europe depuis le déclenchement de la guerre de sécession aux États-Unis.

9 L'ouverture économique marqua le point de départ d'une entreprise de pillage systématique des richesses de l'Égypte et de son peuple. Le système des prêts en fut le principal instrument: il s'agissait, d'une part, des prêts accordés directement aux paysans égyptiens par des usuriers européens, dans un premier temps, puis par les banques de crédit agricole qui s'implantèrent en Égypte après l'apparition, pour la première fois dans l'histoire de ce pays, d'un système de propriété privée de la terre, et d'autre part des prêts concédés aux dirigeants égyptiens à des taux faramineux par les banques étrangères pour financer des projets d'infrastructure tels que les routes, les canaux, les chemins de fer ou le canal de Suez.

Le surendettement de la classe paysanne permit aux sociétés de crédit agricole de s'emparer progressivement des terres des paysans insolvables. À la fin du XIX siècle, plus d'un cinquième des terres agricoles de l'Égypte étaient ainsi passées aux mains de ces sociétés. Quant à l'État égyptien, sa faillite fut consommée en 1876. Ses finances étant assujetties à la Caisse de la Dette internationale, il dut hypothéquer la plus grande part de ses installations commerciales, industrielles et agricoles. Dans le même temps, l'invasion commerciale de l'Occident frappait de plein fouet les commerçants et artisans égyptiens. C'est de la colère de ces derniers, ruinés, et de tous les paysans spoliés de leurs terres que naquit, en 1881-82, la révolte de 'Urâbi avec l'appui de l'armée égyptienne et des intellectuels nationalistes. Cette révolte était l'expression du refus de la pénétration étrangère : il fallait reconquérir la volonté nationale usurpée, autrement dit rendre l'Égypte aux Égyptiens.

11 Ce que la révolte put concrétiser à ses débuts, elle le doit à cette alliance nationale; ce qui, en revanche, précipita sa défaite, ce furent, d'une part, la supériorité technique, industrielle et militaire de l'Occident, et d'autre part la trahison du khédive et de ses auxiliaires, les grands propriétaires terriens. L'occupation britannique vint alors entériner définitivement la subordination de l'Égypte au marché capitaliste mondial et mettre une dernière touche à la liquidation du système étatique que Muhammad 'Ali avait mis en place et de la Nahda qu'il préconisait.

Étant donné le cours que prirent les événements, il n'est guère surprenant que le concept d'État centralisé ait joué un rôle central dans la pensée de la Nahda et dans la réflexion sur les causes respectives de l'essor ou du retard des nations. L'État centralisé fut la clef de voûte de l'entreprise de construction nationale que mena Muhammad 'Ali et qu'Ismâ'îl tenta par la suite de relancer. Tahtâwi, que l'on considère comme le véritable fondateur de la pensée sociopolitique égyptienne contemporaine, rapporte pour sa part la nécessité du centralisme étatique en Égypte à la notion de "société hydraulique » :

«L'Égypte, écrit-il en s'inspirant de Clot Bey, tire du Nil sa fertilité et sa prospérité lorsque le sort d'autres pays est fonction des pluies et des saisons. En cela, l'Égypte plus que toute autre était prédestinée à la réussite, pourvu que son gouvernement fût discipliné et son peuple laborieux, car tout dérèglement dans l'administration de ce pays peut perturber ses cultures. [...] Une forme d'organisation s'imposait. Mais elle ne pouvait venir ni des individus, ni des villages, des bourgs ou des villes, ni même de leur regroupement. Bien plutôt, c'était à un pouvoir central ('umûmiya) qu'elle incombait, car seule l'autorité du gouvernement permettait de mener à bien la réforme de l'agriculture. Ainsi, lorsque l'Égypte adopta le système de l'irrigation 
pérenne et planifiée, il devint indispensable de bien contrôler et coordonner la gestion des eaux, concernant notamment l'assainissement des canaux et la construction des ponts et des barrages. Les formes de pouvoir local (al-hukûma almanziliya) qu'avait connues l'Égypte avaient fait preuve en effet d'une mauvaise organisation qui s'était traduite par une détérioration des services publics: l'absence de gouvernement centralisé en était la cause. » tentative entreprise par Ismâ'îl pour faire renaitre l'État de Muhammad 'Ali et moderniser l'Égypte. Ainsi dégageait-il ici les enseignements que l'on pouvait tirer de l'expérience de Muhammad 'Ali tout en observant celle d'Ismâ'îl.

Quant à Muhammad 'Abduh, lorsqu'il prônait le despotisme éclairé, il plaidait en fait en faveur d'un État fort et centralisé, un État équitable tourné vers le développement, même s'il s'exprimait en d'autres termes et dans un autre contexte idéologique et historique : l'entreprise de la Nahda venait en effet d'avorter du fait de l'occupation britannique, et le temps où les intellectuels philosophaient sur sa conception n'était plus qu'un souvenir.

Quoiqu'il en soit, le concept d'un État fort et centralisé avait donc fait son chemin depuis les débuts de la Nahda dans l'esprit des théoriciens des différentes écoles de pensée : on voyait en lui le moyen de sortir de l'ère du déclin pour entrer enfin dans celle de la Nahda.

Ayant cherché à dégager les concepts fondamentaux de la philosophie de cette "première Nahda», Anouar Abdel Malek a pu établir que «la symbiose étatique » en était l'articulation essentielle :

"Cette symbiose étatique, nous dit-il, découle principalement, mais non toute entière, de l'héritage de la société hydraulique, la plus compacte de toutes il est vrai. Un pouvoir central est toujours objectivement nécessaire pour assurer l'irrigation et les travaux publics qui l'accompagnent. La centralisation, inéluctable, conduit le pouvoir d'État à songer au destin de la terre dont il assume les besoins : cette terre sera donc, des pharaons à Muhammad 'Ali, la propriété exclusive du pouvoir central. Maître des eaux et de la terre, l'État exerce son pouvoir de décision au moyen de deux instruments: l'appareil d'État, et en son cœur l'armée et l'idéologie.»

Toutefois, la nécessité pour l'Égypte d'un État fort et centralisé ne découle pas simplement des exigences de la société hydraulique mais aussi des contraintes de la géographie. Car comme le note Gamâl Hamdân, peu de pays ont vu leur histoire aussi inextricablement liée à leur géographie que l'Égypte.

« Les exigences de la société hydraulique, poursuit Anouar Abdel Malek, ne sont pas les seules en œuvre. C'est que le pays qui subit ces contraintes hydrauliques endogènes est lui-même cerné par une géographie implacable. Situé au carrefour des trois continents qui, jusqu'au siècle dernier, tiennent le destin du monde, au cœur de la plaque tournante qu'est la Méditerranée et le Moyen-Orient, et luimême offrant une surface quasiment étale propice à toutes les pénétrations, sa géographie suscite d'emblée une géopolitique et une position politique de tous les problèmes possibles. D'où encore le rôle de l'État et de l'armée. »

D'une certaine manière, ce déterminisme géographique se traduisait aussi par l'importance de l'élément étranger dans la définition du cours de l'histoire égyptienne. Mais une question se pose alors : la première entreprise de Nahda doit-elle sa faillite aux seules attaques de l'étranger, depuis le Traité de Londres jusqu'à la défaite de 'Urâbi et à l'occupation britannique ? Ou bien ces attaques auraient-elles été infligées à

Égypte/Monde arabe, 26 | 1996 
l'Égypte en raison de certaines fissures internes de l'édifice ? Et si tel fut le cas, quelle pouvait être la nature de ces fissures?

Jusqu'à ce jour, et malgré des tentatives de réponse survenues tardivement, la question est restée en suspens..

Malgré les ténèbres dans lesquelles l'occupation britannique plongea le pays, le peuple égyptien poursuivit la lutte sous la bannière du Parti national tandis que les différents courants de pensée égyptiens persistaient à chercher les moyens de concrétiser le rêve égyptien de la Nahda. Muhammad 'Abduh cherchait à renouveler la pensée religieuse et à réformer l'institution d'al-Azhar en l'ouvrant aux sciences modernes, de même qu'il appelait la réforme du système d'enseignement ; Qâsim Amîn revendiquait la libération de la femme ; Lutfi al-Sayyid lançait le slogan « l'Égypte aux Égyptiens »...

alliance nationale de la même envergure que celle qui avait permis la révolte de 'Urâbi. En effet, à la faveur de la protection temporaire dont jouissait le marché intérieur égyptien depuis que la guerre empêchait l'importation des produits européens, on vit apparaître l'ébauche d'un capitalisme industriel et commercial national - lequel, au demeurant, n'allait pas tarder à être mis en péril dès que la guerre s'arrêterait et que les importations reprendraient. Dans le même temps, les grands propriétaires terriens enregistraient des bénéfices considérables grâce à l'augmentation du prix du coton, ce qui incita certains d'entre eux à s'intéresser à de nouveaux secteurs d'investissement, notamment le commerce et la finance, jusqu'alors entièrement régis par le capital étranger, si bien qu'ils entrèrent momentanément en conflit avec l'occupant.

2 En revanche, la guerre fit supporter au peuple de lourds fardeaux : l'augmentation des prix, la faiblesse des revenus, sans compter l'asservissement subi dans les camps de travail forcé mis en place par les autorités britanniques. qui, sous la conduite des grands propriétaires terriens et des nouveaux capitalistes, leva l'étendard de l'indépendance face à l'occupant et celui de la démocratie face à la famille régnante. La révolution de 1919 laissa sans conteste une profonde empreinte dans la conscience des Égyptiens: elle ancra dans les esprits les notions de nationalisme égyptien, d'unité nationale sans distinction de religion et de laïcité de l'État - «À Dieu la religion, à tous la patrie " - et fit naître un vaste mouvement de renouveau intellectuel et artistique. Mais elle ne réussit en fin de compte à ne réaliser aucun de ses objectifs majeurs.

Concernant la cause nationale, la révolution de 1919, menée par l'oligarchie terrienne, ne conduisit qu'à une indépendance purement formelle car en réalité, après la promulgation de la Constitution de 1923, qui fut la base d'une vie politique très instable, la nouvelle direction politique se contenta de partager le pouvoir avec le palais royal, l'un et l'autre restant assujettis à la domination politique, économique et militaire de l'occupant britannique.

En ce qui concerne l'indépendance économique, aucun changement radical ne survint avec la révolution de 1919: elle s'en tint à une égyptianisation toute partielle qui n'affecta que certains secteurs. Dès avant les années trente, la Banque Misr, créée dans le sillage de la révolution, commença à abandonner la politique qui avait été la sienne au temps de la grande vague de nationalisme, et qui consistait à réserver exclusivement les prises de participation dans ses sociétés au capital égyptien. Aussi, à l'aube des 
années cinquante, l'économie égyptienne était toujours dominée, presque intégralement, par les capitaux étrangers. Les étrangers détenaient en effet plus de la moitié des capitaux des sociétés égyptiennes par actions et occupaient la majorité des sièges de leurs conseils d'administration, tout comme ils contrôlaient les banques, les compagnies d'assurance et les sociétés de commerce international. En outre, les succursales des banques étrangères finançaient l'intégralité de la production cotonnière. Enfin, la Compagnie du canal de Suez se dressait là comme un État dans l'État, contrôlée exclusivement par les capitaux franco-anglais, sans que l'Égypte n'obtienne en retour la moindre part de ses bénéfices... Le rêve de l'indépendance politico-économique semblait toujours aussi inaccessible.

Sur le plan social enfin, la situation se détériora sensiblement, et particulièrement dans les campagnes. La carte de la répartition de la propriété terrienne indique clairement les importantes disparités économiques du secteur agricole à cette époque : 2,6 millions de propriétaires terriens possédaient 2,1 millions de feddan ( 1 feddan $=0,42 \mathrm{ha}$ ) tandis que quelque 159722 propriétaires en possédaient 3,8 millions, ce qui revient à dire que $94 \%$ de l'ensemble des propriétaires détenaient seulement $35 \%$ des terres agricoles alors que les $65 \%$ restants étaient aux mains de $6 \%$ des grands propriétaires.

Le tableau paraît encore plus sombre lorsqu'on sait que les propriétaires terriens ne représentaient qu'une maigre part de la population des campagnes, dont la majorité était composée des ouvriers agricoles et de leur famille. En 1952, ils étaient 11 millions environ et leur revenu annuel moyen ne dépassait pas quatre livres égyptiennes.

La situation n'était guère meilleure dans les villes étant donné la faiblesse du capitalisme égyptien et le retard des structures de production. Le taux de croissance économique restait médiocre, entraînant une progression du chômage et une diminution des salaires.

Dans ces circonstances, il n'est guère étonnant qu'à l'avènement de la deuxième guerre mondiale, avec toutes les épreuves qu'elle fit endurer au peuple et à la classe moyenne sur les plans économique et social, l'Egypte ait connu une période d'instabilité politique et sociale qui se traduisit par un accroissement de la violence politique et par des soulèvements ouvriers et paysans. Mais si le mécontentement social était à l'origine de ces soulèvements populaires, ces derniers étaient dus également à l'incapacité du système en place à régler la question nationaliste. On vit alors apparaître de nouvelles formations politiques qui se mirent à réfléchir aux moyens de sortir de la crise politique et sociale en posant notamment le problème de la propriété terrienne. Une sorte de réforme agraire fut réclamée par l'Association des pionniers (Gamâ'a alruwwâd) avec l'appui timide de l'Union des industries égyptiennes, tandis que les partis communiste et socialiste prônaient la nationalisation des intérêts étrangers. Mais avant toute chose, les problèmes sociaux étaient à l'ordre du jour, car il était entendu que leur résolution était la condition sine qua non du règlement de la question nationale.

Ainsi l'idée s'imposa que pour soustraire l'Égypte à la domination coloniale, il fallait au préalable se débarrasser de celle de l'aristocratie terrienne.

31 Sur la scène de la lutte politique et sociale de l'Égypte, toutes les questions étaient dès lors posées: l'indépendance nationale, la nationalisation des intérêts étrangers, la réforme agraire et enfin l'arabisme, avec lequel l'Égypte renoua en 1948 sur la terre de Palestine. 
C'est alors que survint la Révolution de 1952. Au début de la Révolution, le slogan lancé par Nasser était : « Relève la tête, mon frère ! ». C'était le signe que l'avilissement de la nation allait enfin cesser. L'Égypte ne serait plus un pion sur l'échiquier des luttes politiques internationales : elle avait décidé de descendre dans l'arène et d'assumer son rôle historique dans la région. Mais ce slogan avait également une autre signification, plus importante encore : il appelait le peuple, si longtemps tenu à l'écart, à reprendre sa place au sein de la vie politique et sociale. Il y avait donc là une double revendication: l'indépendance nationale et la justice sociale. Mais que signifiaient réellement ces deux objectifs et quels étaient les moyens d'y accéder?

Nasser se posait en héritier des pionniers du mouvement indépendantiste égyptien, et notamment de Muhammad 'Ali, pour lequel l'indépendance nationale signifiait avant tout la reprise en main des centres de décision politique, sociale, économique et militaire. Voici ce qu'il en disait dans un discours du 21 février 1959 :

«La recherche de la liberté sous toutes ses formes, politique, économique et sociale, ne visait en fait qu'à engendrer une volonté libre, fière et indépendante, qui construirait elle-même son avenir et façonnerait de ses propres mains les lendemains glorieux qu'elle s'était choisis. S'affranchir du colonialisme, mes frères, c'était libérer la volonté indépendante; s'affranchir du féodalisme, c'était aussi libérer la volonté indépendante; et s'affranchir de la domination du pouvoir par le capital, c'était encore libérer la volonté indépendante. Car si la liberté est conquise, et par là même la volonté indépendante, alors la route est toute tracée pour que les peuples puissent mettre en œuvre leur expérience et réaliser leurs vastes desseins et aspirations. "

Puis il poursuivait :

« Toutes ces batailles n'étaient en réalité qu'une seule et même guerre, la guerre d'indépendance. La résistance à la colonisation fut une bataille de la guerre d'indépendance; le renversement du Roi fut une bataille de la guerre d'indépendance; l'abolition du féodalisme fut une bataille de la guerre d'indépendance; la suppression des partis fut une bataille de la guerre d'indépendance; le combat contre le désespoir et l'appel à la confiance et à la foi furent une bataille de la guerre d'indépendance... Toutes ces batailles n'étaient qu'une seule et même guerre. »

L'indépendance et la libération de la volonté nationale, tels étaient, au fond, les objectifs de la réforme agraire qu'imposa la révolution à ses débuts. Car il fallait mettre un terme au pouvoir politique et économique dont jouissait l'aristocratie terrienne, première alliée de l'occupation britannique en Égypte. L'idée était non seulement d'avoir en main la clé de la décision politique, mais aussi de redistribuer plus équitablement le revenu de l'agriculture afin de créer un marché national pour la toute nouvelle production industrielle du pays.

Vint ensuite la lutte pour l'évacuation des troupes britanniques; à peine eut-elle pris fin que lui succéda la prodigieuse bataille pour le haut-barrage et, dans son sillage, la nationalisation du canal de Suez et la défaite de l'agression tripartite en 1956.

La bataille menée pour le haut-barrage révéla que d'ores et déjà, l'Égypte n'était plus prête à se soumettre à l'hégémonie des grandes puissances ni à assujettir son économie au contrôle de la Banque mondiale ou de quelque autre institution. Si les grandes puissances voyaient, dans le financement de la construction du haut-barrage, l'occasion de récupérer l'ascendant qu'elles avaient perdu sur la volonté de l'Égypte, celle-ci allait prouver qu'elle était en mesure de financer cette entreprise grâce à ses propres richesses. C'est ici qu'intervint la nationalisation de la Compagnie du canal de Suez, 
moins de trois ans après l'échec de la nationalisation des pétroles iraniens par Mossadegh.

La bataille de Suez qui s'engagea alors et la victoire sur l'agression tripartite annonça la chute du colonialisme et le début de l'ère des indépendances nationales en Afrique, en Asie et en Amérique Latine. L'Égypte avait sa place sur la scène internationale et jouait avec les grandes puissances sans leur permettre de se jouer d'elle. En outre, la bataille de Suez, qui avait déclenché un vaste mouvement de solidarité arabe, fut une nouvelle occasion pour l'Égypte, après la bataille des pactes militaires et notamment du Pacte de Bagdad, de redécouvrir son arabité et de lutter sur un nouveau terrain. Une étape décisive s'amorça alors pour le mouvement indépendantiste arabe, qui changea la face de la carte politique du monde arabe : ce fut d'abord la révolution irakienne, puis la victoire de la révolution algérienne...

Sur le plan intérieur, la bataille de Suez permit à l'Égypte de reprendre le contrôle de son économie nationale: dès l'arrêt de l'agression tripartite, les intérêts anglais et français furent nationalisés. Ainsi, pour la première fois depuis la fin du règne de Muhammad 'Ali, l'Égypte revint de fait aux Égyptiens... Aucune société n'était plus contrôlée par les Anglais ni par les Français. Les banques et les compagnies d'assurance furent nationalisées à leur tour, de sorte que la propriété publique remplaça le capital étranger dans tous les secteurs de l'économie nationale. C'était le prélude à une reprise en main intégrale des décisions économiques à l'échelle du pays : en 1961, en effet, les grandes nationalisations permirent d'abolir le capitalisme aristocratique égyptien et marqua la naissance du secteur public, qui régit le premier plan de développement économique (1961-1965). Au cours de cette période, l'Égypte enregistra les meilleurs taux de croissance industrielle de son histoire contemporaine.

À l'aube des années soixante, la carte politique, économique et sociale de l'Égypte avait donc subi des modifications radicales. Sur le plan politique, tout d'abord, l'Égypte était devenue le leader incontesté du monde arabe et l'élément actif le plus important du mouvement des non-alignés dans les pays du tiers-monde. Sur le plan économique ensuite, l'État égyptien, fort de la reprise en main intégrale de son économie, s'engagea dans le plus vaste processus de construction nationale indépendante jamais entrepris depuis l'époque de Muhammad 'Ali. Quant au domaine social, enfin, des millions d'ouvriers et de paysans égyptiens purent prendre une part active à la vie de leur pays après en avoir été écartés durant des siècles, et leurs enfants vinrent remplir les rangs des universités, des instituts et des écoles, en réponse aux voeux de Taha Hussein.

41 Cependant, une nouvelle attaque devait survenir. En 1967 commença une campagne de liquidation du nassérisme, à l'intérieur comme à l'extérieur. De même que Muhammad 'Ali avait été éliminé parce qu'il tentait d'instaurer un État puissant sur la route des Indes, Nasser fut tenu en échec parce qu'il tentait de se dresser sur la route du pétrole.

Tout comme l'avait été, en son temps, l'action politique de Muhammad 'Ali, l'expérience nassérienne est aujourd'hui reniée au nom de la liberté du marché ou de la construction de la société civile... Mais ce que l'on veut effacer, en fait, c'est l'État centralisé et son rôle dans le processus de construction nationale indépendante et dans l'entreprise de la Nahda.

43 Cependant la question subsiste : les attaques menées de l'extérieur auraient-elles pu être couronnées d'efficacité sans quelque faille interne? Et dans ce cas, où donc se situait cette faille? 
Les réponses ne manquent pas, mais aucune d'entre elles ne recueille l'unanimité. En tout état de cause, ce qui importe ici, ce ne sont pas les aspects négatifs ni les lacunes : ce qui se passe aujourd'hui, c'est que sous prétexte de combler ces lacunes, l'on fait table rase de tous les aspects positifs de l'expérience nassérienne. Au nom de l'activité du marché, on ouvre la voie à un capitalisme parasitaire et dépendant agissant par l'intermédiaire et sous la botte des grandes sociétés multinationales. Au nom de la liberté du marché, l'on remet en cause tous les acquis des ouvriers et des paysans et l'on abolit le système de la réforme agraire. Au nom de la liberté économique, l'État démissionne de son rôle dans l'éducation et la santé des citoyens.

4 La dette extérieure maintient toujours l'économie égyptienne sous la tutelle de l'étranger, à présent que l'ancienne Caisse de la dette est réapparue sous la forme du Fonds monétaire international. Des millions d'Égyptiens, qu'ils soient paysans ou qu'ils appartiennent à la classe moyenne, se sont vus relégués à nouveau dans leur pauvreté. Et tandis que dans le ciel du Caire s'élevaient des gratte-ciel dont les appartements se vendraient à des millions de livres, les démunis ont pris le chemin des cimetières, à la périphérie du Caire, pour y établir résidence... Toutes choses qui se sont accompagnées d'une virulente campagne de dénigrement du nassérisme et de son chef de file.

L'image de Nasser, pourtant, est toujours présente dans le cœur de ces millions d'Égyptiens, telle celle d'un héros légendaire dont on ne saurait dire au juste s'il a réellement existé ou s'il n'est que le produit de notre imagination, tant son œuvre semble dépasser l'entendement.

«Relève la tête, mon frère!»: le cri nourrit toujours l'esprit de lutte du peuple égyptien, pour que puisse reprendre un jour la bataille immortelle de la Nahda.

\section{INDEX}

Mots-clés : Nahda, Nasser (Gamal Abdel), nationalisme, 'Issa (Hossam), Muhammad Ali 